\title{
The psychosis proneness: psychosis persistence model as an explanation for the association between urbanicity and psychosis
}

\author{
JANNEKE SPAUWEN and JIM VAN OS
}

\begin{abstract}
Given the relatively high attributable risks associated with urbanicity it is crucially important to learn more on how this exposure impacts on population risk for schizophrenia. Further elucidation of the environmental influences that currently go under the name of "urbanicity" therefore is a crucial step in unravelling the etiology of schizophrenia. In order to make the step from association to cause, a plausible mechanism detailing the pathway from impact of exposure to onset of psychotic symptoms is needed. This paper outlines possible avenues and clues to the identification of possible mechanisms and pathways. The focus is on mechanisms of gene-environment interaction in the context of both functional genetic polymorphisms and epigenetic variation.
\end{abstract}

\section{INTRODUCTION}

There may be no other issue in schizophrenia research with more far-reaching public health implications than the finding that young people growing up in an urban environment accumulate an increased risk for schizophrenia (Lewis et al., 1992; Torrey et al., 1997; Mortensen et al., 1999; van Os et al., 2001; Spauwen et al., 2006).

\section{Causality?}

The fact that the incidence of schizophrenia increases consistently with increasing levels of urbanicity in a doseresponse fashion suggests not only statistical association, but also causality (van Os, 2004). It is likely that the kind of geographical variation in incidence associated with urbanization reflects an environmental exposure, and that this environment has its impact, through continuous or repeated exposure, on developing children and adolescents and, possibly, the epigenome (Pedersen \& Mortensen, 2006). Thus, the time window of exposure is not around the time of onset of psychotic disorder, but long before (Marcelis et al., 1999; Pedersen \& Mortensen, 2001a). Recent studies in Germany, Greece, the UK and the Netherlands (van Os et al., 2001; Stefanis et al., 2004; Spauwen et al., 2006) have shown that the increased level of risk for psychotic disorder in urban populations is not

Address for correspondence: Professor J. van Os, Department of Psychiatry and Neuropsychology, Maastricht University, PO BOX 616 (DRT 10), 6200 MD Maastricht (The Netherlands).

Fax: +31-43-3875444

E-mail: j.vanos@ sp.unimaas.nl

Declaration of Interest: None. phenotypically silent, because the prevalence of at-risk mental states, characterized by subtle psychosis-like phenomena, is also higher in urban areas, independent of the increased rate of psychotic disorder, and independent of service use, sociodemographic factors including ethnic group, drug use and size of social network (Pedersen \& Mortensen, 2001a, b; van Os, 2004).

Recent analyses also suggest that the increased risk for clinical and non-clinical expressions of psychosis in urban areas is not mediated by neuropsychological impairment, traffic air pollution, obstetric complications or childhood socioeconomic position (Stefanis et al., 2004). Although viral hypotheses have been put forward to explain associations with urbanicity, household crowding is not a risk factor for schizophrenia (Agerbo et al., 2001) and a hypothesized viral exposure for a rare disorder is arguably difficult to reconcile with the lack of mediation of cognitive variables and with elevated prevalences of subtle psychosis-like experiences of $23 \%$ in the most urban versus $13 \%$ in the most rural areas (van Os et al., 2001). The high prevalence of such at-risk mental states is suggestive of a much more widespread environmental exposure with a cumulative impact over the course of development.

\section{Genetic factors: selection or interaction?}

The urban exposure is unlikely to entirely represent a non-causal epiphenomenon resulting from genetic selection, because changes in urban exposure states during childhood also result in changes in risk for the outcome of adult psychosis (Pedersen \& Mortensen, 2001a). As it is increasingly likely that genetic effects in schizophrenia are to a large degree conditional on the environment and, vice versa, that environmental effects are conditional on 
genetic risk (van Os \& Sham, 2003), gene-environment interactions may also play a role in explaining urban effects. For example, genetic liability may make individuals more susceptible to the risk-increasing effect of the environmental factor associated with urbanicity. If urbanicity acts in co-participation with genetic factors it has been speculated that as far as the environmental component is concerned, the underlying mechanism may be best thought of, in terms of impact on the individual, as the cumulative effects of altered social interactions at the level of the person and possibly also beyond that at the level of the wider social environment such as the neighbourhood (van Os et al., 2005). The further elucidation of the environmental influences that currently go under the name of "urbanicity" is thus a crucial step in unravelling the etiology of schizophrenia.

\section{From proxy exposure to plausible mechanism?}

However, a crucial element necessary for making the step from association to cause is lacking: that of a plausible mechanism detailing the pathway from impact of exposure to onset of psychotic symptoms. Urbanicity is merely a proxy description for some underlying environmental factors in urban areas, and these need to be identified first before further progress can be made (Krabbendam \& van Os, 2005).

\section{Identifying the urbanicity exposure}

One hypothesis regarding risk-increasing mechanisms is the impact of "urban stress". What constitutes urban stress has yet to be validated, however, although stress due to noise is a possible candidate. Direct effects of pollution have been hypothesised but have little biological plausibility. Differences in health behaviours may play a part, but substance misuse has been widely controlled for in studies of the association between schizophrenia and urbanicity.

Remarkably little attention has been paid to other social factors (van Os, 2004). However, aspects of the wider social environment have been shown to affect mental health outcomes, also in relation to psychosis. For example, aspects of the wider social environment such as community level of social fragmentation, social isolation and social inequality may account for variation in the incidence of schizophrenia in small areas (van Os et al., 2000). The impact of such variables may be truly ecological, affecting every person in the community regardless of their individual socio-demographic and other characteristics, thus creating a distributed liability in exposed populations (van Os, 2004).
Work conducted so far has considered the urban environment and the large city as homogeneous entities, but in fact there are important within-city contrasts, the study of which may aid in identifying the factors in the urban environment that act to specifically increase the risk for schizophrenia (Krabbendam \& van Os, 2005). The incidence of schizophrenia, contrary to that of common mental disorders (Weich et al., 2003), is not the same across small areas such as neighbourhoods (Farris \& Dunham, 1939; Hare, 1956a, b; Giggs, 1986). Recent studies have focussed on the developmental effect of variables referred to as "social capital" or "the glue that holds society together" (McKenzie et al., 2002). Cognitive social capital, or aspects of the degree of mutual trust, bonding and safety in neighbourhoods exerts a developmental impact on the mental health of children growing up in these environments (Drukker et al., 2003; 2005; De Silva et al., 2005). If cognitive social capital constitutes one of the mechanisms explaining the association between schizophrenia and the urban environment, it is important to examine how exactly the urban exposure impacts on population expression of psychosis.

\section{How does urbanicity impact on population expression of psychosis?}

Given the relatively high attributable risks associated with urbanicity (Marcelis et al., 1998; Mortensen et al., 1999) it is crucially important to learn more on how this exposure impacts on population risk for schizophrenia. In particular, it is important to assess whether the urban exposure remains largely phenotypically silent with regard to the psychosis outcome in exposed individuals (figure 1A), or whether the exposed, as a group, exhibit a broad shift in clinical and non-clinical expression of psychosis (figure 1B). The former model would suggest that the psychosis phenotype in exposed individuals only emerges if combined with another rare cause for schizophrenia, i.e. urbanicity in itself would not be sufficient to cause expression of psychosis. The latter model, however, would suggest that something in the urban environment is sufficient to facilitate the expression of psychosis, although other causal influences may be needed to reach a sufficiently severe level to attract a diagnosis of schizophrenia.

A recent study on this subject showed that exposed individuals as a group display higher levels of psychosis, suggesting that urbanicity is a sufficient factor to cause non-clinical expression of psychosis (figure 1B) (Spauwen et al., 2004). Exposure to other causal factors may be required to subsequently make the shift to levels of psychosis that are compatible with a diagnosis of 
schizophrenia or other psychotic disorders. These findings are compatible with the impact of environmental variation in cognitive social capital that, in combination with predisposing vulnerability (genetic or environmental), results in onset of psychotic disorder.

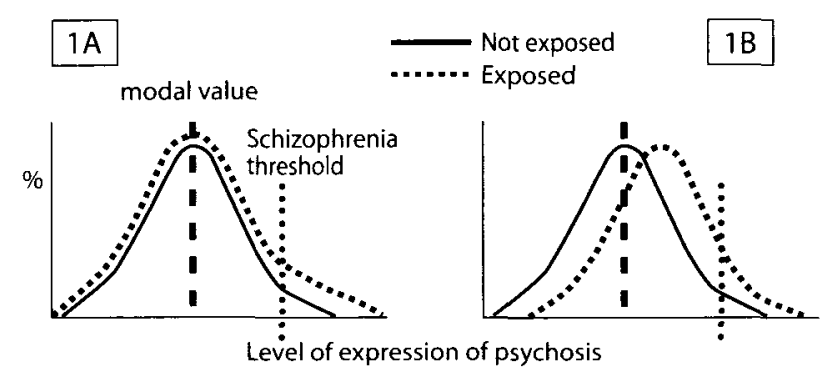

In figure 1A, only a tiny fraction of the exposed population exceeds the level of expression of psychosis compared to the non-exposed, suggesting that the urban exposure is required to combine with a rare causal factor in order to cause the rare disorder schizophrenia. In figure 1B, however, thee exposed, as a group, exhibit a broad shift in clinical and non-clinical expression of psychosis, suggesting that something in the urban environments is sufficient to facilitate the expression of psychosis, although other causal influences may be needed to reach a sufficiently severe level to attract a diagnosis of schizophrenia.

Figure 1. - Impact of urban exposure on population risk.

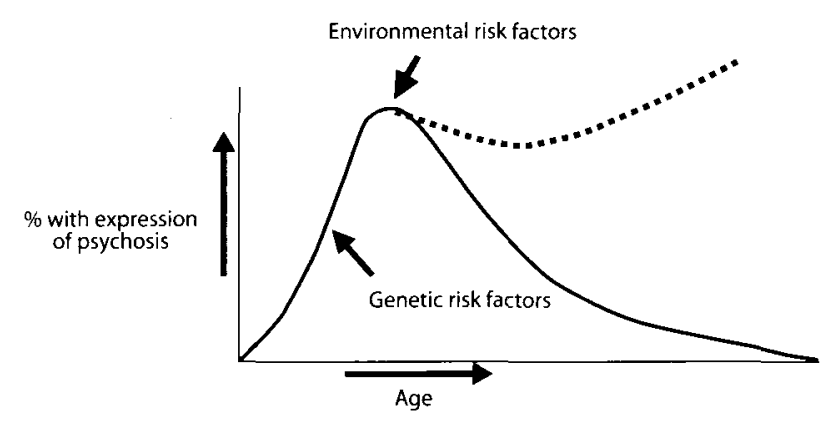

In figure 2 a mechanism is shown whereby the urban environment increases the risk for psychosis. The outcome of the genetically driven and age-associated rise in expression of psychosis associated with puberty persists in an urban environment (environmental risk factors), as shown in the dotted line $2 \mathrm{~A}$, whereas in a rural environment (no environmental risk factor) the expression declines again with age and therefore has a better outcome, solid line $2 \mathrm{~B}$.

Figure 2. - The psychosis proneness - psychosis persistence model.

Thus, a subsequent study showed that the risk-increasing effect of urbanicity on the occurrence of psychotic symptoms was only apparent in those with pre-existing psychosis proneness (or "schizotypy"), independent of other variables that are known to increase the risk for psychosis. This study, using psychometric measures of psychosis proneness, provided a more direct confirmation of previous research (van Os et al., 2003; 2004), where it was found that a proxy genetic risk factor for schizophrenia (a family history of schizophrenia) interacted synergistically with the proxy environmental risk factor that urbanicity represents. To the extent that variation in measures of psychosis-proneness is due to genetic factors (Linney et al., 2003), our findings are compatible with a mechanism of gene-environment interaction. However, to the extent that variation in measures of psychosis proneness is due to environmental factors (van Os et al., 2005) the findings may also represent environment-environment interaction.

\section{The psychosis proneness - psychosis persistence model}

A descriptive mechanism of how the urban environment facilitates the pathway from psychosis proneness to clinical expression of psychotic disorder is the so-called psychosis proneness - psychosis persistence model (Cougnard et al., submitted for publication). This model postulates that the outcome of the age-associated rise in expression of psychosis associated with puberty (Galdos et al., 1993), which is common and can be measured in the general population, is more likely to persist in an urban environment, whereas in a rural environment the expression declines again with age and therefore has a better outcome (figure 2 ). The empirical model thus indicates that the ontogenesis of psychosis may be conceived as the poor outcome of a developmentally common psychosis phenotype (psychosis proneness or schizotypy), which, under the influence of environmental factors in the urban environment, may display abnormal persistence thus possibly increasing the risk for progression towards clinical psychotic disorder (Spauwen et al., 2006).

\section{Possible mechanisms}

\section{Sensitization}

A possible mechanism of risk may reside in behavioural and/or neurochemical sensitization. As discussed above, studies have shown that subjects with a (psychometric or familial) liability for psychosis showed an increased risk for psychotic symptoms or disorder when they were growing up in an urban environment. Living in an urban environment is associated with increased exposure to environmental stress (high traffic densities, neighbourhood noise, violence and victimization, reduced social capital and pollution). The enduring enhancement of the behavioural response to this environmental stress is described as behavioural sensitization (Myin-Germeys et al., 2005). Behavioural sensitization in relation to environmental stress may be interpreted in the light of the dopamine (DA) sensitization hypothesis of psychotic 
symptoms. It has been suggested that schizophrenia is associated with endogenous dopamine sensitization, a state characterized by hyperresponsiveness of DA neurons to environmental stimuli in which even exposure to moderate levels of stress are associated with an excessive DA response (Laruelle, 2000; Kapur, 2003). Furthermore, it has been suggested that dopamine mediates the expression of psychotic experiences from moment-to-moment in patients with schizophrenia (Myin-Germeys et al., 2005).

\section{Social and Cognitive mechanisms}

Individuals with a genetic liability to schizophrenia may be less likely to have their early abnormal mental states corrected in urban areas with lower levels of informal social control, resulting in higher rates of expression of psychosis (van Os et al., 2005) Similarly, aspects of the wider social environment such as lack of perceived safety or social stress might directly contribute to the development of cognitive vulnerabilities for psychosis (Birchwood et al., 2000; Bentall et al., 2001; Garety et al., 2001) resulting in higher rates of psychotic disorder in the genetically predisposed (van Os, 2004).

\section{Gene-urbanicity interactions: from proxy to direct measureres?}

As discussed above, a general mechanism of geneenvironment interaction is supported by replicated population-based evidence suggesting synergism between urbanicity and familial liability in their effect on psychotic disorder (van Os et al., 2003; 2004; Spauwen et al., 2006). Once genes that increase the risk for schizophrenia have been reliably identified, it will be possible to directly investigate genotype-environment interaction. Although four-fifths of the variance in schizophrenia risk is attributable to genes, the locus of a possible genetic defect remains elusive, even given recent optimism (Harrison \& Owen, 2003). The search has been slow and frustrating, probably because there are multiple susceptibility genes (genes that increase the risk for psychiatric disorder, but are neither necessary nor sufficient in themselves to cause illness), each of small effect, which act in conjunction with epigenetic mechanisms and environmental factors.

\section{Functional polymorphisms involved in gene-environment interactions}

One gene involved in gene-environment interactions may be the gene for catechol-O-methyltransferase (COMT) (Caspi et al., 2005; Henquet et al., submitted for publication; Stefanis et al., submitted for publication). The COMT gene codes Catechol-O-Methyltransferase, an enzymatic inactivator of dopamine, norepinephrine and epinephrine. In the prefrontal cortex, COMT is critical in the breakdown of dopamine. The COMT gene, mapped to chromosome $22 q 11$, contains a functional polymorphism, involving a Met to Val substitution at codon 158, which results in two common allelic variants, the Valine (Val) and the Methionine (Met) allele, associated with high versus low enzyme activity (Lotta et al., 1995; Lachman et al., 1996). In the general population, this results in a distribution of individuals with the Met/Met (approximately $25 \%), \mathrm{Val} / \mathrm{Met}(50 \%)$ and $\mathrm{Val} / \mathrm{Val}(25 \%)$ genotype. Increased COMT activity associated with the Val allele may result in a combination of i) reduced dopamine neurotransmission in the prefrontal cortex (Egan et al., 2001), and subsequently ii) increased levels of mesolimbic dopamine signalling, which is hypothesized to predispose to the expression of delusions and hallucinations (Laruelle, 2000; Weinberger et al, 2001; Kapur, 2003). As disturbances in dopaminergic transmission have long been implicated in schizophrenia, and COMT influences frontal lobe function and presynaptic dopaminergic activity, it may provide a possible biological correlate for the association with schizophrenia in conjunction with environmental exposures such as cannabis (Caspi et al., 2005; Henquet et al., submitted for publication), stress (Stefanis et al., submitted for publication) and, possibly, urbanicity. An attractive hypothesis, therefore, is that the cumulative effect of urbanicity interacts with a functional polymorphism such as COMTVal ${ }^{158}$ Met that may be associated with reduced cortical control of subcortical dopaminergic projections, favouring the expression of psychosis (Kapur, 2003; Marcelis et al., 2004; Myin-Germeys et al., 2005).

\section{Epigenetic mechanisms of gene-environment interaction}

Epigenetics is defined as the study of mitotically or meiotically heritable variations in gene function that cannot be explained by changes in DNA sequence (Petronis, 2001). Gottesman \& Bertelsen (1989) examined the risk of schizophrenia in the offspring of MZ twins discordant for schizophrenia. The risks in the offspring of twins did not differ, suggesting that the cause of discordance is not heritable, but rather environmental or epigenetic. Epigenetic mechanisms are only recently being uncovered but it is likely that they are extremely prevalent and affecting many behaviours and health outcomes. Genomic programs could be modified by genetic alterations, which are transmitted in the germ line. For example, Weaver, Meaney and Szyf studied rat maternal care effects on the 
hippocampal transcriptome and anxiety-mediated behaviours in the offspring that are reversible in adulthood. They concluded that "...Our data illustrate a new mechanism by which widespread and stable lifelong interindividual variation in gene expression in the brain might emerge. This mechanism does not require germ-line transmission and could be elicited by natural variations in maternal behaviour early in life". The main difference between genetic and epigenetic variation is the potential for reversal with the appropriate manipulation. Thus, Weaver and colleagues showed that "Our data demonstrate the profound effects that early-life environment might have on the functioning of the genome and its lifelong consequences for behaviour into adulthood' (Weaver et al., 2006).

A recent fascinating study suggests that epigenetic mechanisms may also contribute to the association between urbanicity and schizophrenia, although this explanation was not considered specifically by the authors (Pedersen \& Mortensen, 2006). In this study, it was reported that the increased risk for schizophrenia also applied to children who were born in rural areas, but whose mother had been exposed to an urban environment at the time of birth of the nearest oldest sibling. This data therefore fascinatingly suggest that the effect of urbanicity may consist of silencing or activating genetic material in the germ line that thus "remember" the urban exposure and pass it on to the next generation, regardless of place of birth. Alternatively, the findings may indicate that some of the causes underlying the association between urbanicity and schizophrenia may be rooted in families rather than individuals (Pedersen \& Mortensen, 2006).

\section{REFERENCES}

Agerbo E., Torrey E.F. \& Mortensen P.B. (2001). Household crowding in early adulthood and schizophrenia are unrelated in Denmark: a nested case-control study. Schizophrenia Research 47, 243-246.

Bentall R.P., Corcoran R., Howard R., Blackwood N. \& Kinderman P. (2001). Persecutory delusions: a review and theoretical integration. Clinical Psychology Review 21, 1143-1192.

Birchwood M., Meaden A., Trower P., Gilbert P. \& Plaistow J. (2000). The power and omnipotence of voices: subordination and entrapment by voices and significant others. Psychological Medicine 30, 337-344.

Caspi A., Moffitt T.E., Cannon M., McClay J., Murray R., Harrington H., Taylor A., Arseneault L., Williams B., Braithwaite A., Poulton R. \& Craig I.W. (2005). Moderation of the effect of adolescent-onset cannabis use on adult psychosis by a functional polymorphism in the catechol-O-methyltransferase gene: longitudinal evidence of a gene $\mathrm{X}$ environment interaction. Biological Psychiatry 57, 1117-1127.

Cougnard A., Marcelis M., Myin-Germeys I., de Graaf R., Ten Have M., Krabbendam L., Lieb R., Wittchen H-U, Henquet C., Spauwen J., Verdoux H. \& van Os J. (submitted for publication). Does normal developmental expression of psychosis combine with environmental load to cause abnormal psychosis persistence? A psychosis proneness - psychosis persistence model.
De Silva M. J., McKenzie K., Harpham T. \& Huttly S. R. (2005). Social capital and mental illness: a systematic review. Journal of Epidemiology and Community Health 59, 619-627.

Drukker M., Kaplan C.D., Feron F.J.M. \& van Os J. (2003). Children's health-related quality of life, neighbourhood socio-economic deprivation and social capital. A contextual analysis. Social Science and Medicine 57, 825-841.

Drukker M., Buka S.L., Kaplan C., McKenzie K. \& van Os J. (2005). Social capital and young adolescents' perceived health in different sociocultural settings. Social Science and Medicine 61, 185-198.

Egan M.F., Goldberg T.E., Kolachana B.S., Callicott J.H., Mazzanti C.M., Straub R.E., Goldman D. \& Weinberger D.R. (2001). Effect of COMT Val108/158 Met genotype on frontal lobe function and risk for schizophrenia. Proceedings of the National Academy of Sciences of the United States of America 98, 6917-6922.

Farris R.E. \& Dunham H.W. (1939). Mental Disorders in Urban Areas. University of Chicago Press: Chicago.

Galdos P.M., van Os J.J. \& Murray R.M. (1993). Puberty and the onset of psychosis. Schizophrenia Research 10, 7-14.

Garety P.A., Kuipers E., Fowler D., Freeman D. \& Bebbington P.E (2001). A cognitive model of the positive symptoms of psychosis. Psychological Medicine 31, 189-195.

Giggs J.A. (1986). Mental disorders and ecological structure in Nottingham. Social Science and Medicine 23, 945-961.

Gottesman I.I. \& Bertelsen A. (1989). Confirming unexpressed genotypes for schizophrenia. Risks in the offspring of Fischer's Danish identical and fraternal discordant twins. Archives of General Psychiatry 46, 867-872.

Hare E.H. (1956a). Family setting and the urban distribution of schizophrenia. Journal of Mental Science 102, 753-760.

Hare E.H. (1956b). Mental illness and social conditions in Bristol. Journal of Mental Science 102, 349-357.

Harrison P.J. \& Owen M.J. (2003). Genes for schizophrenia? Recent findings and their pathophysiological implications. Lancet 361, 417-419.

Henquet C., Krabbendam M., Papiol S., Fananás L., Drukker M. Johannes J., Ramaekers P. \& van Os J. (submitted for publication). An experimental study of catechol-O-methyltransferase (COMT) Val158Met moderation of $\Delta$-9-tetrahydrocannabinol-induced effects on psychosis and cognition.

Kapur S. (2003). Psychosis as a state of aberrant salience: a framework linking biology, phenomenology, and pharmacology in schizophrenia. American Journal of Psychiatry 160, 13-23.

Krabbendam L. \& van Os J. (2005). Schizophrenia and urbanicity: a major environmental influence-conditional on genetic risk Schizophrenia Bulletin 31, 795-799.

Lachman H.M., Papolos D.F., Saito T., Yu Y.M., Szumlanski C.L. \& Weinshilboum R.M. (1996). Human catechol-O-methyltransferase pharmacogenetics: description of a functional polymorphism and its potential application to neuropsychiatric disorders. Pharmacogenetics 6, 243-250.

Laruelle M. (2000). The role of endogenous sensitization in the pathophysiology of schizophrenia: implications from recent brain imaging studies. Brain Research. Brain Research Reviews 31, 371-384.

Lewis G., David A., Andreasson S. \& Allebeck P. (1992). Schizophrenia and city life. Lancet $340,137-140$.

Linney Y.M., Murray R.M., Peters E.R., MacDonald A.M., Rijsdijk F. \& Sham P. C. (2003). A quantitative genetic analysis of schizotypal personality traits. Psychological Medicine 33, 803-816.

Lotta T., Vidgren J., Tilgmann C., Ulmanen I., Melen K., Julkunen I. \& Taskinen J. (1995). Kinetics of human soluble and membrane-bound catechol O-methyltransferase: a revised mechanism and description of the thermolabile variant of the enzyme. Biochemistry 34,4202 4210.

Marcelis M., van Os J., Sham P., Jones P., Gilvarry C., Cannon M., McKenzie K. \& Murray R. (1998). Obstetric complications and familial morbid risk of psychiatric disorders. American Journal of Medical Genetics 81, 29-36. 
Marcelis M., Takei N. \& van Os J. (1999). Urbanization and risk for schizophrenia: does the effect operate before or around the time of illness onset? Psychological Medicine 29, 1197-1203.

Marcelis M., Cavalier E., Gielen J., Delespaul P. \& van Os J. (2004). Abnormal response to metabolic stress in schizophrenia: marker of vulnerability or acquired sensitization? Psychological Medicine 34, 1103-1111.

McKenzie K., Whitley R. \& Weich S. (2002). Social capital and mental health. British Journal of Psychiatry 181, 280-283.

Mortensen P.B., Pedersen C B., Westergaard T., Wohlfahrt J., Ewald H., Mors O., Andersen P. K. \& Melbye M. (1999). Effects of family history and place and season of birth on the risk of schizophrenia. New England Journal of Medicine 340, 603-608.

Myin-Germeys I., Delespaul P. \& van Os J. (2005). Behavioural sensitization to daily life stress in psychosis. Psychological Medicine 35, 733-741.

Pedersen C B. \& Mortense, P B. (2001a). Evidence of a dose-response relationship between urbanicity during upbringing and schizophrenia risk. Archives of General Psychiatry 58, 1039-1046.

Pedersen C.B. \& Mortensen P.B. (2001b). Family history, place and season of birth as risk factors for schizophrenia in Denmark: a replication and reanalysis. British Journal of Psychiatry 179, 46-52.

Pedersen C.B. \& Mortensen P.B. (2006). Are the cause(s) responsible for urban-rural differences in schizophrenia risk rooted in families or in individuals? American Journal of Epidemiology 163, 971-978.

Petronis A. (2001). Human morbid genetics revisited: relevance of epigenetics. Trends in Genetics TIG 17, 142-146.

Spauwen J., Krabbendam L., Lieb R., Wittchen H.-U. \& van Os J. (2004). Does urbanicity shift the population expression of psychosis? Journal of Psychiatric Research 38, 613-618.

Spauwen J., Krabbendam L., Lieb R., Wittchen H.-U. \& van Os J. (2006). Evidence that the outcome of developmental expression of psychosis is worse for adolescents growing up in an urban environment. Psychological Medicine 36, 407-415.

Stefanis N., Delespaul P., Smyrnis N., Lembesi A., Avramopoulos D., Evdokimidis I., Stefanis C. \& van Os J. (2004). Is the excess risk of psychosis-like experiences in urban areas attributable to altered cognitive development? Social Psychiatry and Psychiatric Epidemiology 39, 364-368.
Stefanis N.C., Henquet C., Avramopoulos D.A., Smyrnis N., Evdokimidis I.K., Myin-Germeys I., Stefanis C.N. \& van Os J. (submitted for publication). COMT Val158Met moderation of stress-induced psychosis.

Torrey E.F., Bowler A.E. \& Clark K. (1997). Urban birth and residence as risk factors for psychoses: an analysis of 1880 data. Schizophrenia Research 25, 169-176.

van Os J. (2004). Does the urban environment cause psychosis? British Journal of Psychiatry 184, 287 - 288.

van Os J. \& Sham P. (2003). Gene-environment correlation and interaction in schizophrenia. In The Epidemiology of Schizophrenia (ed. R.M. Murray, P.B. Jones, E. Susser, J. van Os and M. Cannon). Cambridge University Press: Cambridge.

van Os, J., Driessen G., Gunther N. \& Delespaul P. (2000). Neighbourhood variation in incidence of schizophrenia. Evidence for person-environment interaction. British Journal of Psychiatry 176, 243-248.

van Os J., Hanssen M., Bijl R.V. \& Vollebergh W. (2001). Prevalence of psychotic disorder and community level of psychotic symptoms: an urban-rural comparison. Archives of General Psychiatry 58, 663-668.

van Os J., Hanssen M., Bak M., Bijl R.V. \& Vollebergh W. (2003). Do urbanicity and familial liability coparticipate in causing psychosis? American Journal of Psychiatry 160, 477-482.

van Os, J., Pedersen C.B. \& Mortensen P. B. (2004). Confirmation of synergy between urbanicity and familial liability in the causation of psychosis. American Journal of Psychiatry 161, 2312-2314.

van Os J., Krabbendam L., Myin-Germeys I. \& Delespaul P. (2005). The schizophrenia envirome. Current Opinion in Psychiatry 18, 141-145.

Weaver I.C., Meaney M.J. \& Szyf M. (2006). Maternal care effects on the hippocampal transcriptome and anxiety-mediated behaviors in the offspring that are reversible in adulthood. Proceedings of the National Academy of Sciences of the United States of America 103, 3480-3485.

Weich S., Holt G., Twigg L., Jones K. \& Lewis G. (2003). Geographic variation in the prevalence of common mental disorders in britain: a multilevel investigation. American Journal of Epidemiology 157, 730-737.

Weinberger D.R., Egan M.F., Bertolino A., Callicott J.H., Mattay V.S., Lipska B.K., Berman K.F. \& Goldberg T.E. (2001). Prefrontal neurons and the genetics of schizophrenia. Biological Psychiatry 50, 825-844. 